\title{
Recent Advances in Long Wavelength Quantum Dot Lasers and Amplifiers
}

\author{
R. Nötzel, E. A. J. M. Bente, M. K. Smit, H. J. S. Dorren \\ COBRA Research Institute, Eindhoven University of Technology, $5600 \mathrm{MB}$ Eindhoven, The Netherlands \\ e-mail: r.noetzel@tue.nl
}

\begin{abstract}
We demonstrate 1.55- $\mu \mathrm{m}$ InAs/InGaAsP/InP (100) quantum dot (QD) shallow and deep etched Fabry-Pérot and ring lasers, micro-ring lasers, mode-locked lasers, Butt-joint integrated lasers, polarization control of gain, and wavelength conversion in QD amplifiers.

OCIS codes: (250.5960) Semiconductor lasers; (250.5980) Semiconductor optical amplifiers; (310.6628) Nanostructures
\end{abstract}

\section{Intoduction}

We review our recent advances in long wavelength quantum dot (QD) lasers and amplifiers. Focus is on the InAs/InGaAsP/InP (100) materials system as the materials system of choice for $1.55-\mu \mathrm{m}$ wavelength telecom devices and their monolithic integration. Topics covered include wavelength tuning of InAs/InGaAsP/InP (100) QDs grown by metal organic vapor phase epitaxy (MOVPE), shallow and deep etched Fabry-Pérot (FP) and ring lasers, micro-ring lasers, mode-locked lasers, Butt-joint integrated extended cavity lasers, polarization control of gain of semiconductor optical amplifiers (SOAs), and multi-wavelength conversion in SOAs. We will not address work on quantum dashes or wires, other substrate orientations, and the InAs/GaAs materials system.

\section{Wavelength tuning}

When InAs QDs are grown by MOVPE on quaternary InGaAsP layers (in our case lattice-matched InGaAsP with bandgap at $1.25 \mu \mathrm{m}, \mathrm{Q} 1.25)$, their emission wavelength at room temperature (RT) is far beyond $1.6 \mu \mathrm{m}$ due to As/P exchange, rendering the QDs too big. We have solved this problem by the insertion of ultra-thin (1-2 monolayers) GaAs interlayers underneath the QDs. The GaAs interlayers passivate the Q1.25 surface against As/P exchange. Hence, upon increase of the interlayer thickness the QD height is continuously reduced and the emission wavelength tuned through the $1.55 \mu \mathrm{m}$ wavelength range [1]. The QD density is $2-3 \times 10^{10} \mathrm{~cm}^{-2}$.

\section{Shallow and deep etched FP and ring lasers}

The narrow-ridge waveguide lasers with active region of five-fold stacked InAs QD layers operate in continuous wave (CW) mode at RT on the QD ground state (GS) transition. Device parameters for as-cleaved shallow etched FP lasers with $3.5-\mu \mathrm{m}$ waveguide width are: $125 \mathrm{~mA}$ threshold current for 1-mm cavity length, $6 \mathrm{~A} / \mathrm{cm}^{2}$ transparency current density per QD layer, $4.2 \mathrm{~cm}^{-1}$ internal optical loss, $37 \%$ internal differential quantum efficiency, $15 \mathrm{~cm}^{-1}$ maximum QD GS modal gain, and $80 \mathrm{~nm} 3 \mathrm{~dB}$ gain bandwidth [2]. The deep etched FP lasers with $1.65 \mu \mathrm{m}$ waveguide width exhibit the same threshold current as shallow etched lasers due to the zerodimensional carrier confinement inhibiting non-radiative sidewall recombination (Fig. 1). The broad lasing spectrum reflects the inhomogeneous nature of the QD gain medium. The low absorption of the QDs allows for unpumped output waveguides of the 2-mm circumference QD ring lasers, increasing the flexibility for photonic integration [3]. CW lasing at RT has been reported also in Refs. 4 and 5, however, on the QD excited state (ES) transition.
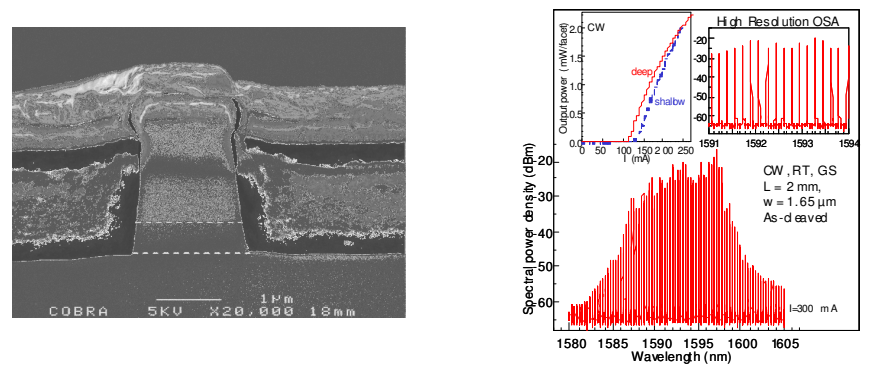

Fig. 1. Left: Cross-sectional scanning electron microscope (SEM) image of the deep etched FP QD laser. Right: Lasing spectrum at 300 mA. Insets: Light-current characteristics of shallow and deep etched FP QD lasers and lasing spectrum with enhanced resolution. 


\section{OWJ5.pdf}

\section{Micro-ring lasers}

Electrically pumped micro-ring QD lasers operate in CW mode at RT for diameters down to $22 \mu \mathrm{m}$ for which the threshold current is $12.5 \mathrm{~mA}$ [6]. The small bending radii are allowed by deep etching the $2-\mu \mathrm{m}$ wide waveguides, enabled by the QD gain medium (Fig. 2).
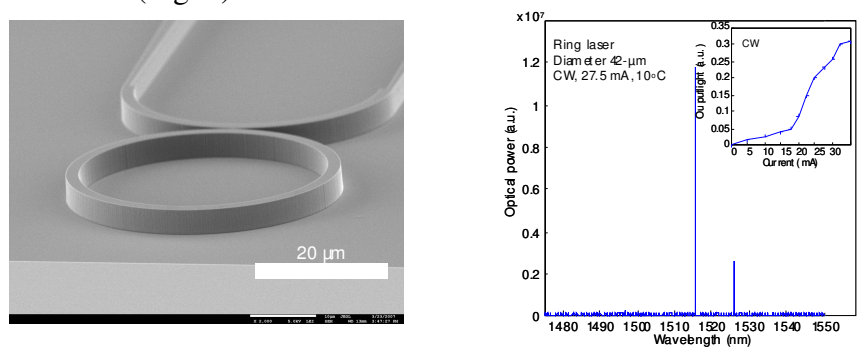

Fig. 2. Left: SEM image of the micro-ring QD laser after waveguide etching. Right: Lasing spectrum of the $42-\mu \mathrm{m}$ diameter ring laser at 27.5 $\mathrm{mA}$. Inset: Light-current characteristics showing a threshold current of $16 \mathrm{~mA}$.

\section{Mode-locked laser}

Passive mode-locking is achieved in two-section QD lasers (Fig. 3). The output pulses are heavily up-chirped with a value of $20 \mathrm{ps} / \mathrm{nm}$ [7]. The output spectrum is coherent over $10 \mathrm{~nm}$. Stable mode locking is observed in a much wider parameter field of forward current in the SOA section and reverse bias of the absorber section compared to mode-locked lasers with bulk active region.
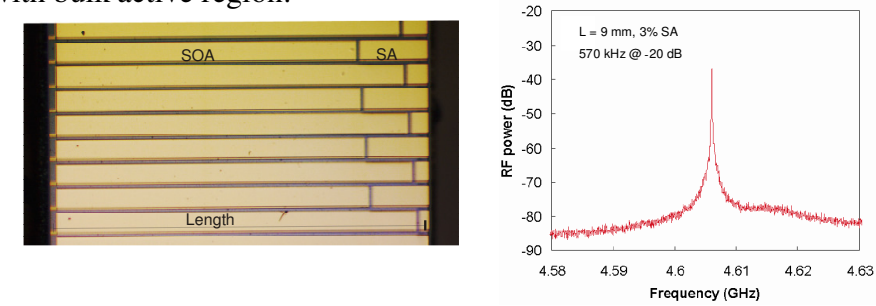

Fig. 3. Left: Photograph of various two-section mode-locked QD lasers. Right: RF-spectrum for a $9 \mathrm{~mm}$ device with $3 \%$ saturable absorber (SA) length. Injection current is $900 \mathrm{~mA}$ and SA bias voltage is - $1 \mathrm{~V}$. The RF-peak is $43 \mathrm{~dB}$ over the noise floor and the width is $570 \mathrm{kHz}$ at $-20 \mathrm{~dB}$.

\section{Butt-joint integrated extended cavity laser}

Operation of a monolitically integrated extended cavity laser in CW mode at RT on the QD GS transition demonstrates the compatibility of our QD gain material with Butt-joint active-passive integration technology (Fig. 4) [8]. The threshold current is comparable to that of our all-active FP QD lasers. The Butt-joint reflectivity is below $-40 \mathrm{~dB}$ for straight waveguides.
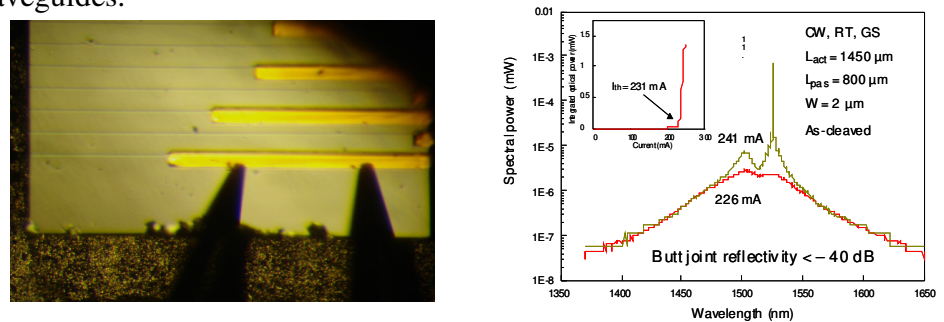

Fig. 4. Left: Photograph of various integrated extended cavity QD lasers. The two current probes contact the laser with $1450 \mu \mathrm{m}$ long active and $800 \mu \mathrm{m}$ long passive waveguides. Right: Electroluminescence and lasing spectra as a function of current. Inset: Light-current characteristics.

\section{Polarization control of gain}

Linear polarization control of the cleaved-side emission [9] and gain [10] is achieved for SOAs employing closely stacked InAs QDs with separation layer thicknesses of 4-5 nm. Increasing the number of stacked QD layers to 5 
rotates the linear polarization of the emission and QD GS gain from TE to TM due to vertical electronic coupling. With increase of the injection current the polarization rotates back to TE due to QD ES gain (Fig. 5). This provides novel functionalities such as polarization switching at operation wavelengths between QD GS and ES. TM dominant gain has been reported also in Ref. 11, however, with strongly tensile strained thin separation layers.
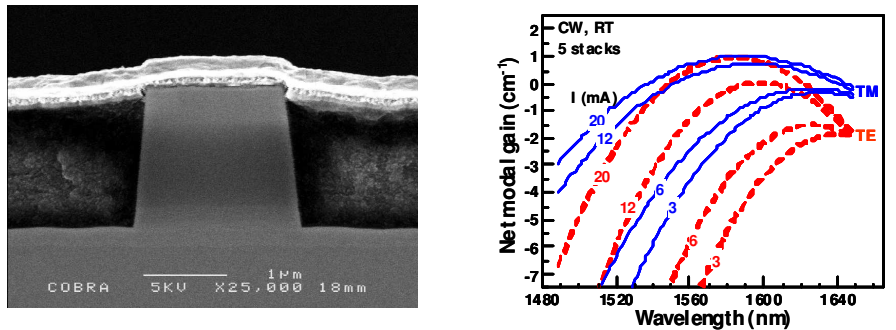

Fig. 5. Left: Cross-sectional SEM image of the QD SOA. Right: TE and TM polarized net modal gain spectra at RT for various CW injection currents.

\section{Wavelength conversion}

Direct non-inverted, error-free, and low penalty $(<2.5 \mathrm{~dB})$ multi-wavelength conversion at $40 \mathrm{Gbit} / \mathrm{s}$ is demonstrated in a QD SOA based on a simplified filtering scheme (Fig. 6) [12]. By detuning the filter pass band to the shorter wavelength, the QD SOA induced phase modulation is converted into amplitude modulation. Non-inverted wavelength conversion is realized by the enhanced blue chirped components of the output pulses. Wavelength conversion has been reported also in Ref. 13 via four-wave mixing and a small-signal modulation bandwidth $>25$ $\mathrm{GHz}$ has been suggested.
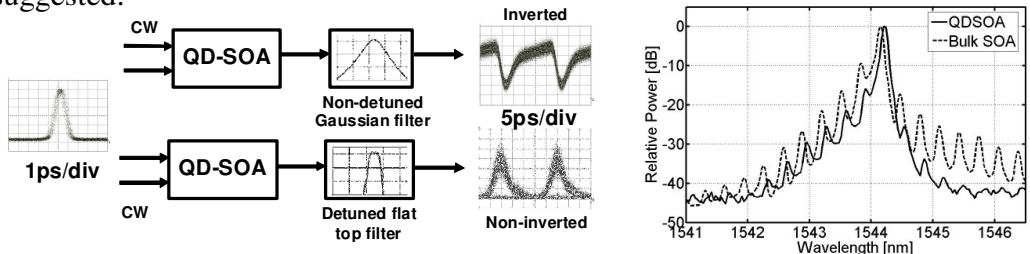

Fig. 6: Left: Schematic operation with a non-detuned and detuned filter (top and bottom) giving inverted or non-inverted signal. Left: Spectrum at output of the QD SOA (solid) compared to that of a bulk SOA (dashed).

\section{References}

[1] S. Anantathanasarn, R. Nötzel, P. J. van Veldhoven, T. J. Eijkemans, and J. H. Wolter, "Wavelength tunable (1.55 $\mu \mathrm{m}$ region) InAs quantum dots in InGaAsP/InP (100) grown by metal organic vapor phase epitaxy,” J. Appl. Phys. 98, 013503-1-7 (2005).

[2] S. Anantathanasarn, R. Nötzel, P. J. van Veldhoven, et al., "Lasing of wavelength-tunable InAs/InGaAsP/InP (100) quantum dots grown by metal organic vapor phase epitaxy," Appl. Phys. Lett. 89, 073115-1-3 (2006).

[3] Y. Barbarin, S. Anantathanasarn, E. A. J. M. Bente, Y. S. Oei, M. K. Smit, and R. Nötzel, “1.55- $\mu \mathrm{m}$ range InAs-InP (100) quantum-dot Fabry-Pérot and ring lasers using narrow deeply etched ridge waveguides," IEEE Phot. Technol. Lett. 18, 2644-2646 (2006).

[4] H. D. Kim, W. G. Jeong, J. H. Lee, J. S. Yim, D. Lee, R. Stevenson, P. D. Dapkus, J. W. Jang, and S. H. Pyun, "Continuous-wave operation of $1.5 \mu \mathrm{m}$ InGaAs/InGaAsP/InP quantum dot lasers at room temperature," Appl. Phys. Lett. 87, 083110-1-3 (2005).

[5] D. Franke, M. Moehrle, J. Boettcher, P. Harde, A. Sigmund, and H. Kuenzel, "Effect of metal organic vapor phase epitaxy growth conditions on emission wavelength stability of 1.55 $\mu$ m quantum dot lasers," Appl. Phys. Lett. 91, 081117-1-3 (2007).

[6] M. T. Hill, S. Anantathanasarn, Y. Zhu, Y. S. Oei, P. J. van Veldhoven, M. K. Smit, and R. Nötzel, "InAs-InP (1.55- $\mu$ m region) quantum-dot microring lasers," IEEE. Phot. Technol. Lett. 20, 446-448 (2008).

[7] M. J. R Heck, E. A. J. M. Bente, E. Smalbrugge, Y. S. Oei, M. K. Smit, S. Anantathanasarn, and R. Nötzel, "Observation of Q-switching and mode-locking in two-section InAs-InP quantum dot lasers at 1.53 um," Optics Express, 15, 16292-16301 (2007).

[8] H. Wang, J. Yuan, P. J. van Veldhoven, et al., "Butt joint integrated extended cavity InAs/InP (100) quantum dot laser emitting around 1.55 $\mu \mathrm{m}$, , IEE Electron. Lett. 44, 522-523 (2008).

[9] S. Anantathanasarn, R. Nötzel, P. J. van Veldhoven, F. W. M. van Otten, T. J. Eijkemans, and J. H. Wolter, "Stacking and polarization control of wavelength-tunable (1.55- $\mu \mathrm{m}$ region) InAs/InGaAsP/InP (100) quantum dots," Appl. Phys. Lett. 88, 063105-1-3 (2006).

[10] S. Anantathanasarn, P. J. van Veldhoven, T. J. Eijkemans, et al., "Polarization control of gain of stacked InAs/InP (100) quantum dots at $1.55 \mu \mathrm{m}$ : Interplay between ground and excited state transitions," Appl. Phys. Lett. 92, 123113-1-3 (2008).

[11] T. Akiyama, M. Sugawara, and Y. Arakawa, "Quantum-dot semiconductor optical amplifiers," Proc. IEEE 95, 1757-1766 (2007).

[12] O. Raz, J. Herrera, N. Calabretta, E. Tangdiongga, S. Anantathanasarn, R. Nötzel, and H. J. S. Dorren, "Non-inverted multiple wavelength converter at $40 \mathrm{Gbit} / \mathrm{s}$ using $1550 \mathrm{~nm}$ quantum dot SOA," Electron. Lett. 44, 988-989 (2008).

[13] D. Nielsen, S. L. Chuang, N. J. Kim, D. Lee, S. H. Pyun, W. G. Jeong, C. Y. Chen, and T. S. Lay, "High-speed wavelength conversion in quantum dot and quantum well semiconductor optical amplifiers," Appl. Phys. Lett. 92, 211101-1-3 (2008). 